\title{
Estrategia de mejora del liderazgo directivo y las habilidades gerenciales en el nivel educativo superior
}

\author{
Herminia Katty Muñoz Iparraguirre \\ https://orcid.org/0000-0002-0701-2771 \\ Universidad San Ignacio de Loyola, Lima, Perú \\ Walter Vicente Tuesta Luna \\ johaotuestam@gmail.com \\ https://orcid.org/0000-0001-9601-9440 \\ Universidad San Ignacio de Loyola, Lima, Perú \\ Fernando Alexis Nolazco Labajos \\ https://orcid.org/0000-0001-8910-222X \\ Universidad San Ignacio de Loyola, Lima, Perú \\ Jaime David Menacho Carhuamaca \\ https://orcid.org/0000-0002-2496-4280 \\ Universidad Cesar Vallejo, Lima, Perú
}

\section{RESUMEN}

El objetivo del presente estudio consistió en analizar la relevancia de la estrategia de mejora del liderazgo directivo y las habilidades gerenciales en el ámbito educacional superior con el propósito de fortalecer el perfil y las competencias directivas. Se tuvo un estudio mix to y contó con un muestreo por conveniencia la participación de directivos y coordinadores en el nivel superior a quienes se les pudo entrevistar, asimismo se aplicó una encuesta validada. Los resultados evidenciaron la regularidad de las habilidades gerenciales de los directivos. Por lo tanto, se concluyó en la imperiosa necesidad de elaborar una propuesta de fortalecimiento de las estrategias de liderazgo directivo para la mejora de las habilidades gerenciales y blandas para la revaloración de la convivencia laboral y el buen trato en una organización educativa.

Palabras clave: habilidades gerenciales; habilidades humanas; liderazgo directivo; perfil directivo; estrategia de mejora. 


\title{
Strategy for improving executive leadership and managerial skills at the higher education level
}

\begin{abstract}
The objective of this study was to analyze the relevance of the strategy to improve managerial leadership and managerial skills in higher education in order to strengthen the managerial profile and competencies. There was a mixed study and a sampling by convenience included the participation of managers and coordinators at the higher level who could be interviewed, a validated survey was also applied. The results showed the regularity of the managerial skills of the directors. Therefore, it was concluded in the urgent need to elaborate a proposal to strengthen the management leadership strategies for the improvement of managerial and soft skills for the reassessment of work coexistence and good treatment in an educational organization.
\end{abstract}

Keywords: managerial skills; human skills; executive leadership; managerial profile; improvement strategy

Artículo recibido: 05 octubre. 2021 Aceptado para publicación: 02 noviembre 2021 Correspondencia: johaotuestam@gmail.com Conflictos de Interés: Ninguna que declarar 


\section{INTRODUCCIÓN}

Las reformas curriculares acontecidas en los últimos años tanto a nivel local como mundial han permitido cambios educacionales sustantivos en los procesos de gestión institucional y pedagógico. Los avances acelerados en materia tecnológica y científica bajo un contexto de competitividad e innovacion, se encuentran en una continua transformación direccionada a la mejora de la calidad educativa con criterios de exigencia, pertinencia y credibilidad organizacional (Organización de Estados Iberoamericanos, 2017; Weinstein y Muñoz, 2019). En ese sentido, la sociedad actual requiere una cadena de valor que evidencie la predisposición efectiva de las prácticas y competencias profesionales de los responsables en la conducción de las escuelas y las instituciones de enseñanza superior. Por ello, es indispensable que cumplan de forma óptima las principales funciones de liderazgo y gestión educacional, valorando el recurso más significativo que constituyen los trabajadores que forman parte del talento humano organizacional (Romero, 2021; Bolívar, 2019)

Del mismo modo, las instituciones educativas enmarcadas en un contexto de innovacion tecnológica de la enseñanza tienden a implementar un plan estratégico que les permita la consolidación de la confianza, el liderazgo efectivo que se proyecte más allá del entorno organizacional (Córdova, Rojas y Marín, 2021). Asi, los cambios exigen prácticas de liderazgo adecuadas por parte de los directivos en base al desarrollo de las habilidades gerenciales que promuevan una gestión educativa y asuman un rol protagónico tanto en la convivencia laboral, los procesos pedagógicos e institucional que lleven a la mejora de la enseñanza y la calidad educativa (López y López, 2019; Sardón, 2017). Por lo tanto, el propósito del artículo es analizar la relevancia de una estrategia de mejora del liderazgo directivo y las habilidades gerenciales en el nivel educativo superior. Asimismo, pretende contribuir de forma académica al incremento significativo del marco teórico con una propuesta de impacto a la mejora respectiva del perfil directivo, las prácticas de liderazgo educacional y las habilidades gerenciales

Al abordar lo sustentado por Ferreira (2021) entorno al liderazgo de la gestión educacional en instituciones de enseñanza foráneas, es fundamental generar las condiciones saludables para promover un entorno laboral positivo entre los integrantes de la organización escolar con el propósito efectivo de la mejora de los procesos de gestión y el desarrollo de los aprendizajes. Tambien, es relevante acotar que la aplicabilidad de 
las habilidades de liderazgo gerencial permitirá evidenciar la calidad educativa entorno al proceso de enseñanza aprendizaje, lo que conlleva al fortalecimiento de las competencias profesionales, actitudinales de los directivos y docentes desde una perspectiva holística proactiva. (Romero y Krichesky, 2018)

Es interesante la propuesta investigada por Mestanza y Vega (2021) entorno al liderazgo directivo en una organización escolar y los acuerdos que pueda arribar con los docentes para la mejora continua de los procesos de gestión educacional, para evitar que un estilo de conducción asumido por los directivos en vez de optimizarlo lo deteriore. De esta manera, es indispensable que se promueva una convivencia adecuada, de respeto mutuo que permitirá gestionar las acciones institucionales y pedagógicas de manera pertinente. Por ello, se ha coincidido con Barrientos y Alania (2021); Fuentes y Pérez (2019) en considerar el clima escolar como el factor delimitante para que las practicas de liderazgo educacional sean efectivas y es responsabilidad de los directivos generar las condiciones para su desarrollo en la institucion educativa. Tambien, es pertinente considerar que las habilidades de liderazgo directivo deben estar contextualizadas a la realidad problemática escolar que incluya los enfoques de inclusión, equidad de género e interculturalidad para la mejora de los procesos de gestiona institucional (Roemintoyo, Tauhid, Miyono y Kamil Budiarto, 2021; Niebla e Ibarra, 2017)

El liderazgo directivo se fundamenta en la propuesta teórica del desarrollo organizacional y su base de apoyo en la planificación desde una perspectiva multidisciplinaria. De esta manera, los directivos y responsables de la conducción institucional tienden a formular y programar estrategias viables que promuevan el empleo de conductas y comportamientos acordes a la realidad organizacional con el propósito de realzar las fortalezas, actitudes y destrezas elementales para el desarrollo de los procesos de gestión institucional. (Madrigal, 2006; Huerta y Rodríguez, 2006). Por otro lado, los que dirigen una entidad son conscientes de la importancia de desarrollar conocimientos y habilidades específicas entorno a las organizaciones, asi como de las actitudes predispuestas al manejo responsable de las habilidades blandas con los integrantes de la organización para conseguir mejorar en los procesos de calidad y excelencia institucional (Gallardo e Ibarra, 2021; Anderson, 2012; Kohn y James, 2000)

Por otro lado, las relaciones humanas han afianzado la preocupación e interés por la convivencia organizacional. Así, Mayo (2004) pudo identificar que el agotamiento y 
cansancio laboral influye en el desempeño de los trabajadores, y por ende en las actividades institucionales. En ese sentido, es relevante resaltar el papel que desempeña el talento humano en una organización, asi como las relaciones de convivencia para el pertinente trabajo direccionados con las motivaciones que cada uno experimenta. El percibir un salario no es solo el propósito del trabajador sino alcanzar otros beneficios que ayudarán en su crecimiento laboral y debe partir de los directivos fortalecer sus competencias. Los integrantes de una institución asumen los retos y desafíos de mejora continua de forma colaborativa para consolidar un optimo desempeño en el cumplimiento de los propósitos organizacionales (Blanch, 2003). Del mismo modo, la presencia de la escuela humanista ha permitido afianzar la relevancia del individuo como un elemento indispensable en los procesos organizacionales, puesto que direcciona la gestión entorno al comportamiento y desenvolvimiento de las personas. Es por ello que es basico la formulacion de estrategias de liderazgo que alcance la transformación de las habilidades gerenciales para consolidar el impacto hacia la comunidad (Diaz, 2013)

En el ámbito educativo, el liderazgo directivo es definido como aquel accionar que tiende a influir en los demás con el propósito de mejora de los aprendizajes y debe forjarse contando con el apoyo desinteresado de los integrantes de una institucion, mediante la reflexión metódica de las acciones efectuadas por los directivos que permitan la apropiada gestión educacional y el proceso de toma de decisiones para la mejora continua institucional (Bolívar, 2019). En cuanto a las habilidades gerenciales son aquellas destrezas practicas y procedimientos que son indispensables para el desempeño optimo y eficiente de la función directiva, estas pueden ser índole técnica, conceptual, el trato individual y colectivo (Katz, 1974).

Es preciso acotar que el perfil idóneo del directivo requiere un conjunto variado de competencias, destrezas y experiencias ajustados a las exigencias del contexto educativo en el que desempeña su liderazgo, aunado con los conocimientos solidos para evidenciar la eficacia y competencia a fin de que ejecute sus actividades específicas (Dery, 2021; Díaz, Espinoza, Peterson y Kuri, 2019). Un directivo reúne las competencias adecuadas cuando predispone sus habilidades para el buen funcionamiento y rendimiento de cada uno de los recursos con los que cuenta la organización, logrando un impacto significativo y cuyos resultados se evidenciaran en el logro efectivo de las metas educativas organizacionales (Bolívar, 2019; Pérez, 2021) 
Por lo tanto, liderazgo directivo es clave para gestionar el desarrollo efectivo colaborativo de las diversas instancias, áreas o ámbitos institucionales con las que interactúa de forma pertinente. La presencia de un líder que monopoliza las acciones y pretende resolverlo todo sin ayuda, tiende a direccionarse al fracaso, puesto que las metas y propósitos no podrán efectuarse (Medina y Gómez, 2014). Así, un líder con una visión y determinación, que practique la empatía, adecúe su nivel de comunicación y sepa dar y recibir retroalimentación evidenciara a mediano y largo plazo una transformación paulatina de los procesos de gestión de la organización educativa

\section{METODOLOGÍA}

Se ha predispuesto una investigación mixta, puesto que abordó información cualitativa y cuantitativa contando con el apoyo de las entrevistas y el cuestionario para el recojo de la información valedera. Se contó con la participación de directivos, coordinadores académicos del nivel superior y mediante el muestreo por conveniencia se predispuso en cuarenta participantes a quienes se le aplicó un cuestionario. Del mismo modo, se consideró cuatro participantes con más de diez años de experiencia directiva a quienes se le pudo entrevistar mediante una video conferencia individualizada. El procedimiento efectuado estuvo direccionado al recojo de las percepciones de los participantes que fueron triangulados contando con el apoyo de los programas estadísticos que sirvió para la obtención de los primeros hallazgos relevantes (Carhuancho, Nolazco, Sicheri, Guerrero y Casana, 2019; Hernández y Mendoza, 2018; Rodríguez y Pérez, 2017)

\section{RESULTADOS}

Los resultados obtenidos del proceso de triangulacion de la información y del análisis de los datos recogidos de la encuesta fueron debidamente clasificadas y permitió identificar en el caso del estudio cualitativo la categoría emergente.

En el caso de los resultados cuantitativos, el nivel regular de significancia de las habilidades gerenciales se ha predispuesto en el trato personal y de trabajo en equipo (90\% de los encuestados) por encima de las habilidades técnicas (60\%) y conceptuales $(57,5 \%)$ quedando el nivel de eficiencia como un reto de mejora institucional en el buen trato y la participacion de los integrantes de la comunidad educativa. 
Figura 1. Nivel de habilidades de trato personal y de trabajo en equipo

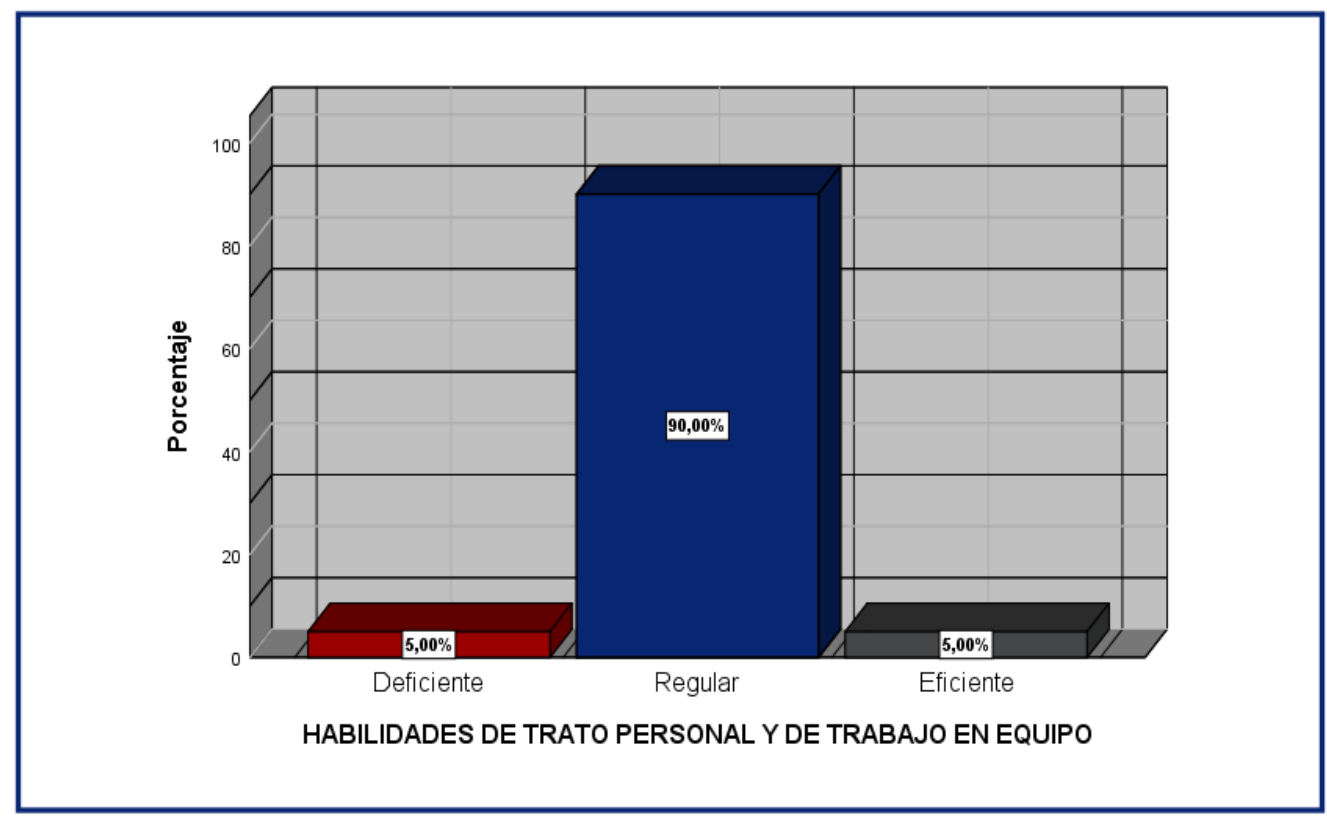

A diferencia de las otras habilidades, se evidencia en los resultados que el trato personal como parte de la convivencia institucional es regular y el nivel de eficiencia es de 5\% similar al deficiente, lo que conlleva a una evaluación organizacional de este aspecto indispensable.

Por otro lado, las habilidades gerenciales muestran un nivel regular $(67,5 \%)$ en contraste con el eficiente (30\%) y el deficiente (2,5\%), lo que evidencia la necesidad de la presencia de un liderazgo directivo para afrontar el cambio hacia la eficiencia

\section{Figura 2. Nivel de habilidades gerenciales}

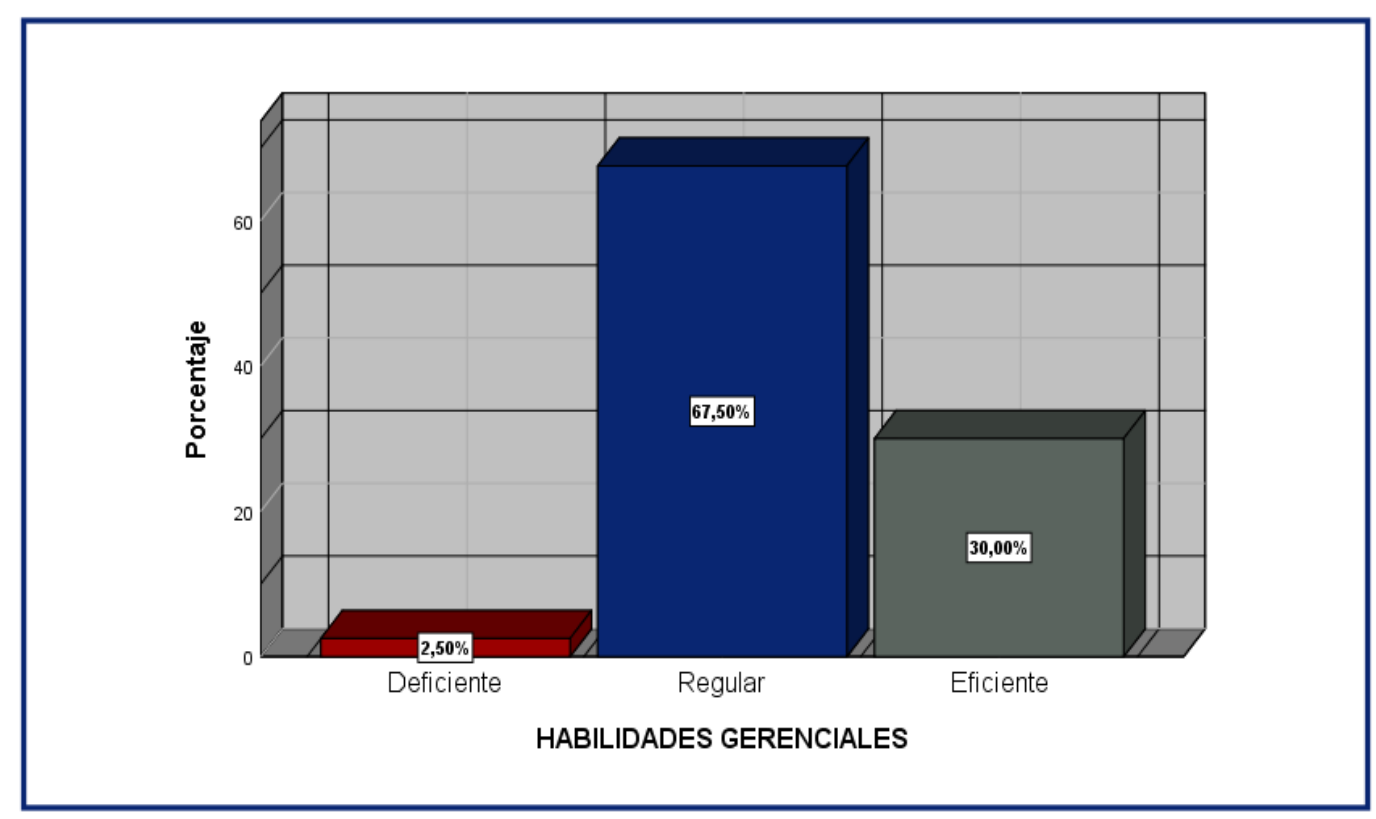


En el caso de los resultados cualitativos, es relevante considerar que el trato personal y de trabajo en equipo se circunscribió entorno al autocontrol, autoconfianza, equilibrio emocional, empatía, y la presencia emergente del perfil directivo para ocupar el puesto respectivo tal como se visualiza en la figura 3. Sin lugar a dudas, los entrevistados consideraron que la inadecuada toma de decisiones en el proceso de gestión institucional producto de la inestabilidad emocional generara un escenario enrarecido y por ello es fundamental actuar con prestancia y el manejo efectivo de las habilidades blandas durante la convivencia institucional.

El liderazgo aparece de forma emergente, puesto que su práctica pertinente permitirá fortalecer las habilidades gerenciales del directivo para que puedan brindar soporte e implementacion de las competencias y talento del personal a su cargo. Es por ello que el conocimiento y valoración de los trabajadores permitirá identificar sus cualidades y fortalezas tal como se visualiza en la figura 4. La ausencia de liderazgo imposibilitará las conexiones institucionales, el trabajo en equipo, asi como la transformación institucional en perjuicio de los estudiantes.

Las habilidades gerenciales son parte esencial y requisito indispensable para acceder al puesto directivo, debido que permitirá el optimo desempeño organizacional. En ese sentido, los resultados evidenciaron que el establecimiento de la convivencia laboral, la conformación del trabajo en equipo podrá criterios orientados al cumplimiento de los propósitos educacionales. La adecuada gestión no afectará la toma de decisiones pertinentes, ni influirá la inestabilidad emocional de los directivos 


\section{Figura 3.}

Análisis de las habilidades de trato personal y de trabajo en equipo.

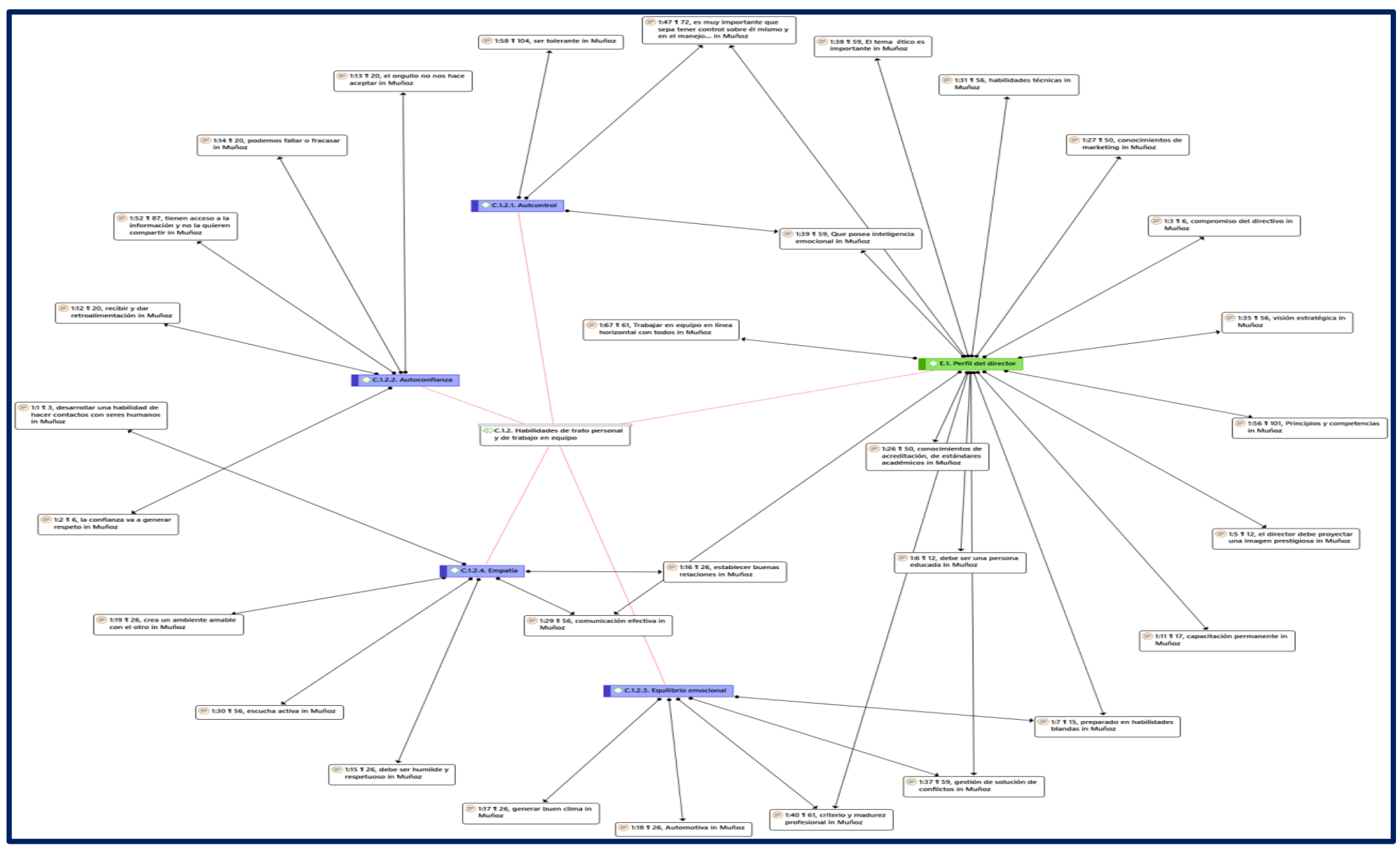

Ciencia Latina Revista Científica Multidisciplinar, Ciudad de México, México.

ISN 2707-2207 / ISSN 2707-2215 (en línea), noviembre-diciembre, 2021, Volumen 5, Número 6. https://doi.org/10.37811/cl_rcm.v5i6.1262 p12469 


\section{Figura 4.}

Análisis de la categoría emergente liderazgo

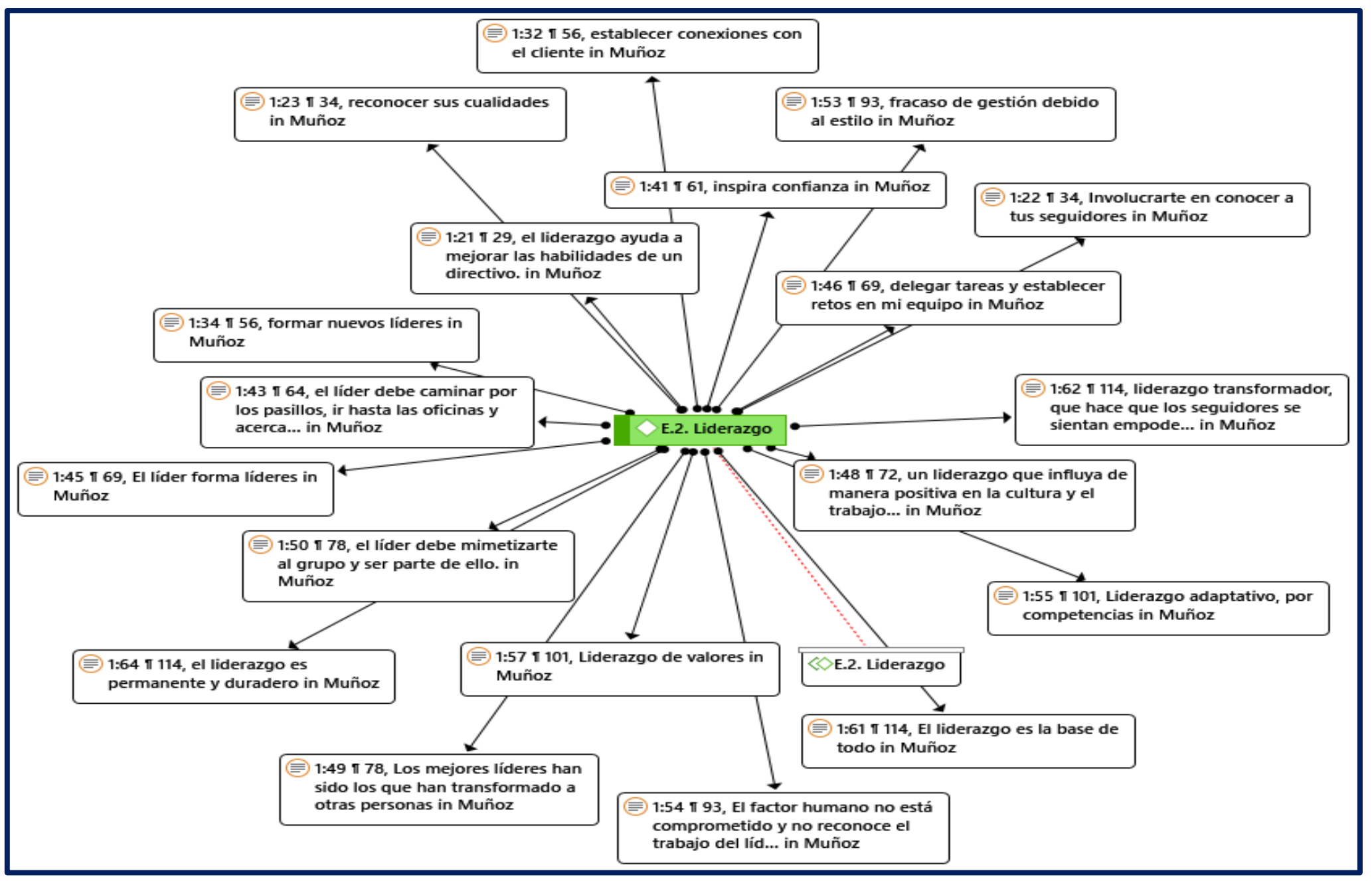

Ciencia Latina Revista Científica Multidisciplinar, Ciudad de México, México.

ISN 2707-2207 / ISSN 2707-2215 (en línea), noviembre-diciembre, 2021, Volumen 5, Número 6 https://doi.org/10.37811/cl_rcm.v5i6.1262 p12470 


\section{Figura 5.}

Análisis de las habilidades gerenciales

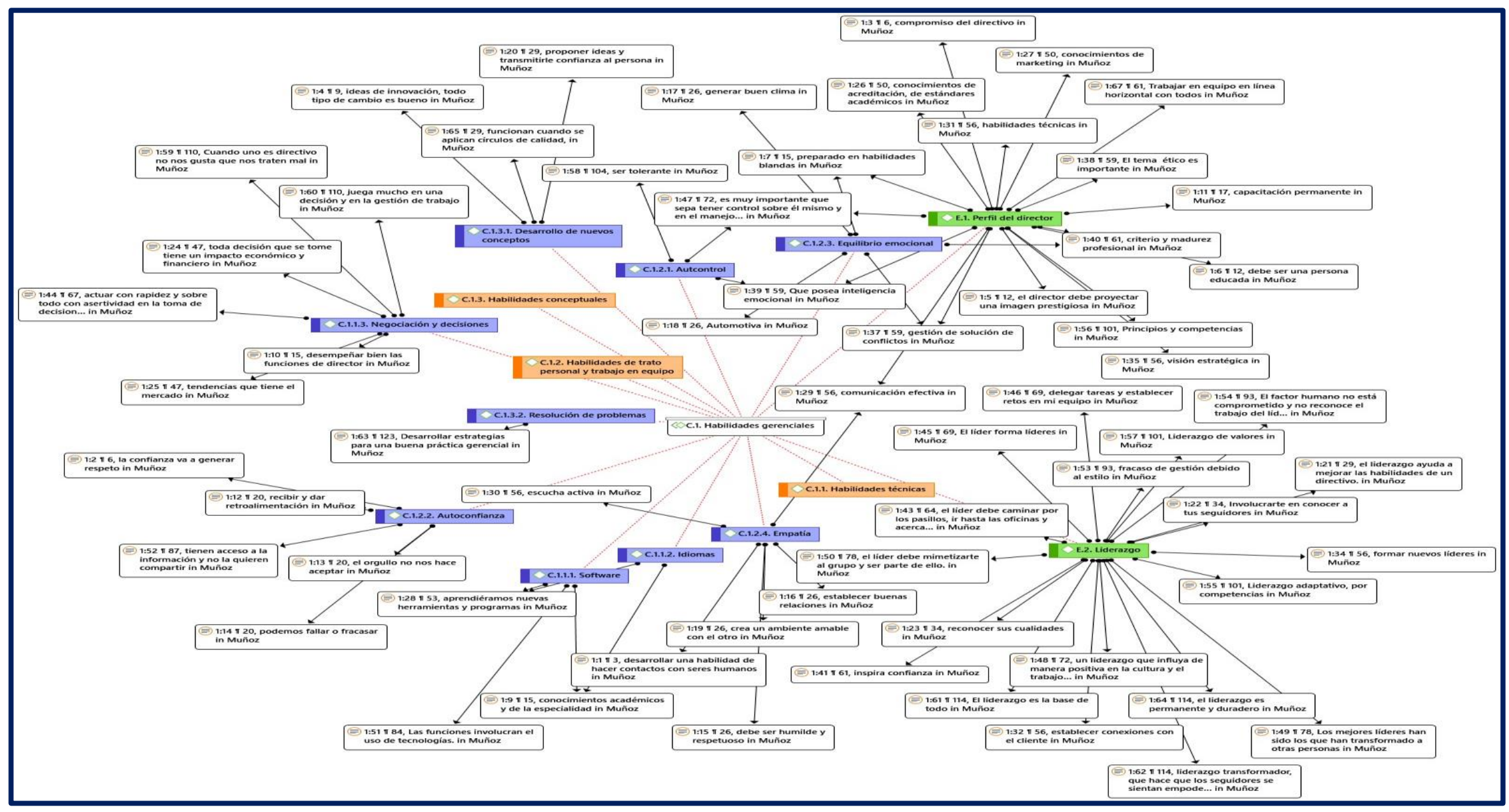

Ciencia Latina Revista Científica Multidisciplinar, Ciudad de México, México.

ISN 2707-2207 / ISSN 2707-2215 (en línea), noviembre-diciembre, 2021, Volumen 5, Número 6.

https://doi.org/10.37811/cl_rcm.v5i6.1262 p12471 


\section{Discusión}

El estudio evidenció la relevancia del liderazgo directivo, la formulacion de estrategias pertinentes para su efectividad en la mejora de las habilidades gerenciales en el nivel de enseñanza superior. Esta situación ameritó que los directivos fortalezcan sus competencias profesionales, liderazgo para que los trabajadores que integran una organización educacional participen en los procesos de gestión de manera conjunta. Sin embargo, la realidad ha mostrado que el nivel regular de las habilidades gerenciales, el trato personal y de trabajo en equipo por encima de la eficiencia.

Los resultados obtenidos, se asocian con Mestanza y Vega (2021) en que la presencia de liderazgo es fundamental en los procesos de gestión y en el perfil directivo de mejora de las habilidades gerenciales. De esta manera, la identificación y valoración de la labor del personal asignado contribuirá a la percepción democrática y equitativa de la toma de decisiones institucionales, evitando la presencia de un estilo verticalista de conducción que deteriore la convivencia organizacional. Así, es relevante la promoción de las actitudes y habilidades blandas para que las actividades organizacionales se efectúen de manera armoniosa.

En cuanto a las habilidades gerenciales y los resultados obtenidos, se ha coincidido con la investigación de Romero y Krichesky (2018) entorno a la aplicabilidad del trato personal y el trabajo en equipo como referente indispensable de liderazgo directivo, permitirá evidenciar la mejora de los procesos de gestión institucional y por ende la calidad educativa de la enseñanza en el nivel superior. Del mismo modo, la perspectiva se direccionó en relación al desarrollo de las competencias profesionales y actitudinales para poder afianzar la mejora organizacional respectiva, puesto que los hallazgos han mostrado que la regularidad constituye un resultado alto a contraparte de la eficiencia, lo que conlleva a la predisposición del cambio respectivo.

Por otro lado, se ha coincidido con la propuesta de Bolívar (2019) quien manifestó la importancia del perfil idóneo del directivo educacional. En ese sentido, se pudo evidenciar en los resultados que las prácticas de liderazgo es la base de todo accionar institucional y los entrevistados manifestaron que la presencia de un liderazgo transformador fortalecerá el trabajo en equipo, valorando el desempeño del personal que labora en la organización educativa. Así, las competencias directivas permitirán el buen funcionamiento institucional para consolidar y mejorar los procesos de gestión. 
Se puede sustentar que, de acuerdo a los resultados evidenciados, se asocia con López y López (2019) en cuanto los entrevistados pudieron identificar las habilidades pertinentes de un líder educacional, mediante acciones especificas como el mimetizarse con el grupo y evitar el desconocimiento contextual. Tambien, es importante acotar que los valores y las actitudes constituyen referentes claves para generar espacios de convivencia adecuada y los integrantes de la organización educacional asuman un rol protagónico en los procesos de gestión institucional y académica.

\section{CONCLUSIÓN}

El propósito de la investigación fue analizar la relevancia de una estrategia de mejora del liderazgo directivo y las habilidades gerenciales en el nivel educativo superior. En ese sentido, la investigación evidenció la presencia de tres problemáticas específicas que ameritan ser atendidas en prontitud, la habilidad regular del trato personal y trabajo en equipo, el inadecuado perfil directivo que se manifiesta en un liderazgo poco efectivo en el manejo del talento humano organizacional.

Asimismo, el estudio evidenció la imperiosa necesidad de implementar las habilidades gerenciales y las estrategias que requieren las personas que asuman la función directiva con el propósito de consolidar un optimo desenvolvimiento en los procesos de gestión. Del mismo modo, es importante señalar que en el entorno educacional se percibe que las habilidades del directivo se encuentran predispuestas en concordancia a sus competencias y actitudes profesionales para el manejo administrativo, y en cuanto al talento humano, debido a su complejidad requiere de fortalecimiento, especialización continua profesional. También, es indispensable la elaboración de una propuesta de fortalecimiento de las estrategias de liderazgo directivo para la mejora de las habilidades gerenciales Por consiguiente, las futuras investigaciones pueden desarrollar las temáticas abordadas desde una perspectiva sistémica, puesto que los estudios de mayor impacto se evidencian en la formulacion de planes efectivos y viables para generar una solución adecuada a la problemática identificada en el proceso de investigación.

\section{REFERENCIAS BIBLIOGRAFICAS}

Anderson, D. (2012). Organization development (2nd ed.). Sage

Blanch, J. (2003). La Escuela de las Relaciones Humanas en Teoría de las Relaciones Laborales. Editorial UOC 
Barrientos, D., \& Alania, R. (2021). Liderazgo directivo y clima institucional: radiografía de instituciones educativas privadas del sur oriental del Perú. Paidagogo, 3(1), 128-149. https://doi.org/10.52936/p.v3i1.50

Bolívar, A. (2019). Una dirección escolar con capacidad de liderazgo pedagógico. La Muralla

Carhuancho, I., Nolazco, F., Sicheri, L., Guerrero, M., \& Casana, K. (2019). Metodología para la investigación holística. UIDE

Córdova, E., Rojas, I., \& Marín, S. (2021). El liderazgo directivo de las instituciones educativas: una revisión bibliográfica. Conrado, 17(80), 231-236. http://scielo.sld.cu/scielo.php?script=sci_arttext\&pid=S1990$86442021000300231 \& \operatorname{lng}=$ es\&tlng=pt.

Dery, K. (2021). The Ideological Perspective of School Principals' PerceptionsEducational Leaders Defining their Roles. European Journal of Educational Management, 1(4), 13-23. https://doi.org/10.12973/eujem.4.1.13

Díaz E., Espinoza J., Peterson H. \& Kuri I. (2019). Una propuesta de evaluación de las competencias de liderazgo transformacional en los estudiantes de licenciatura, Diálogos sobre Educación. Temas actuales en investigación educativa, 10 (19), 1-18. https://doi.org/10.32870/dse.v0i19.615

Diaz, S. (2013). Lo humano en la Teoría de las Organizaciones. Visión Gerencial, 1, 4557. https://www.redalyc.org/pdf/4655/465545895008.pdf

Fuentes, L. S., \& Pérez, L. M. (2019). Convivencia escolar: una mirada desde las familias. Telos: Revista de Estudios Interdisciplinarios en Ciencias Sociales, 21(1), 61-85. https://dialnet.unirioja.es/servlet/articulo?codigo=6773126

Gallardo, L., \& Ibarra, C. (2021). Habilidades directivas y académicas de coordinadores de programas educativos universitarios basados en competencia. Revista de Investigación latinoamericana en competitividad organizacional RILCO, 9, 6779. https://dialnet.unirioja.es/servlet/articulo?codigo=7879450

Hernández, R., \& Mendoza, C. (2018). Metodología de la investigación. Las rutas cuantitativas, cualitativas y mixtas. Editorial Mc Graw Hill Education

Huerta, J., \& Rodríguez, G. (2006). Habilidades directivas. Pearson Educación 
López, I., \& López, M. (2019). El rol de la gestión directiva en los resultados educativos. Revistas $\quad$ Espacios, $\quad$ 40(36), http://www.revistaespacios.com/a19v40n36/a19v40n36p03.pdf

Ferreira, R. (2021). El liderazgo directivo en la gestión escolar. Comparación de casos. Ciencia Latina Revista Científica Multidisciplinar,5(5), 7219-7238. https://doi.org/10.37811/cl_rcm.v5i5.841

Katz, R. (1974). Habilidades directivas básicas. Worker

Kohn, F. B., \& James, M. A. (2000). Changing the change agent: Marketing the organizational development professional. Journal of Professional Services Marketing, 21(1), 143. https://doi.org/10.1300/J090v21n01_10

Madrigal, B. (2006) Habilidades Directivas. Mc Graw Hill

Mayo, E. (2004). The human problems of an industrial civilization. Routledge

Medina, A. \& Gómez, R. (2014). El liderazgo pedagógico: competencias necesarias para desarrollar un programa de mejora en un centro de educación secundaria. Perspectiva Educacional, Formación de Profesores, 53(1), 91-113. https://www.redalyc.org/articulo.oa?id=333329700007

Mestanza, J., \& Vega, M. (2021). Liderazgo directivo y su impacto en la gestión centrada en los aprendizajes. Revista Epistemia, 5(1), 1-11. https://doi.org/10.26495/re.v5i1.1890

Niebla, S., \& Ibarra, E. (2017). Liderazgo directivo femenino y el clima organizacional en una escuela de educación media superior. Praxis Investigativa ReDIE, 9(17), 42-52. http://repositorio.unsm.edu.pe/handle/11458/2816

Organización de Estados Iberoamericanos (2017). Miradas sobre la Educación en Iberoamérica. OEI

Pérez, E. (2021). Philippine Women Educators' Leadership Styles and Managerial Skills. Revista europea en línea de ciencias naturales y sociales, 10(3), 438-455. https://european-science.com/eojnss/article/view/625

Roemintoyo, R., Tauhid, H., Miyono, N., \& Kamil Budiarto, M. (2021). Contribution Factors in Affecting Teacher Performance: Principal Managerial Skills and Work Motivation. Journal of education technology, 5(2), 220-227. http://dx.doi.org/10.23887/jet.v5i2.34687 
Rodríguez, A., \& Pérez, A. (2017). Métodos científicos de indagación y de construcción del conocimiento. Revista EAN, (82), 179-200. http://www.scielo.org.co/pdf/ean/n82/0120-8160-ean-82-00179.pdf

Romero, C. (2021). Liderazgo Directivo en Escuelas que Superan las Barreras del Contexto. REICE: Revista Iberoamericana sobre Calidad, Eficacia y Cambio en Educación, 19(1), 73-90. https://doi.org/10.15366/reice2021.19.1.005

Romero, C., \& Krichesky, G. J. (2018). Interactive leadership in turbulent school climates. An exploratory study of high school principals from the city of Buenos Aires. Educational Management Administration \& Leadership, 26(2), 339-354. https://doi.org/10.1177/1741143217720456

Sardón, A. (2017). Liderazgo transformacional y la gestión escolar en instituciones educativas primarias. Revista de Investigaciones Altoandinas, 19(3), 295-304. https://doi.org/10.18271/ria.2017.294

Weinstein, J., \& Muñoz, G. (2019). Liderazgo en escuelas de alta complejidad sociocultural: Diez miradas. Ediciones Universidad Diego Portales 